\title{
Preference and information about the time and the occurrence of shock delivery
}

\author{
W. R. SAFARJAN and M. R. D'AMATO \\ Rutgers University, New Brunswick, New Jersey 08903
}

\begin{abstract}
Rats given the option to choose between a signaled-shock condition, in which all signals were followed by shock, and one in which extra signals occurred showed no preference for either condition. In contrast, when all signals were followed by shock, rats showed a strong preference for a condition in which shock followed the signal by a fixed, rather than a variable, time duration. The latter result indicates that some types of information about aversive events may be reinforcing.
\end{abstract}

Recent evidence indicates that preference for signaled over unsignaled inescapable shock is importantly controlled, not by the signal that precedes shock, but by stimuli accompanying the signal's absence (discriminable shock-free time or "safe" periods). However, there are conflicting data regarding the role of the signal as a predictor of shock (Arabian \& Desiderato, 1975; Badia, Harsh, Coker, \& Abbott, 1976). Part of this discrepancy could stem from the fact that stimuli which reliably predict shock contain at least two different types of information. The presence of the signal indicates that shock will occur, while the usual fixed temporal relationship between onset of the signal and onset of shock indicates something about when shock will occur. Badia et al. (1976) manipulated the first source of information by varying the proportion of extra signals and found preference for the signaled over the unsignaled condition to be largely independent of this variable. Arabian and Desiderato (1975), on the other hand, manipulated the second source and found preference for a condition containing less, rather than more, accurate information about the time of shock delivery (but see Collier, 1977). The present research assessed the ability of the two sources of information described above to support preference behavior in a situation where the signal's absence always identified a period free from shock. Thus, any differences in observed responding could be attributed to the reinforcing effects of the signal-shock relationship rather than to stimuli associated with the signal's absence.

\section{METHOD}

\section{Subjects}

Eight naive male albino rats of the Sprague-Dawley strain (Madison, Wisconsin), 90-120 days old at the start of the study, served as subjects. They were maintained under a 12-h light-dark

Supported by NSF Grant BNS7310232. Reprints may be obtained from M. R. D'Amato, Department of Psychology, Rutgers University, Busch Campus, New Brunswick, New Jersey 08903. cycle with free access to food and water. To prevent unauthorized avoidance of shock by rolling on their backs, each animal's back was shaved weekly.

\section{Apparatus}

Subjects were tested in a standard two-lever operant chamber (Grason-Stadler Model 1111-P), which was housed in a soundand light-attenuating enclosure. A Plexiglas ceiling was inserted $3.4 \mathrm{~cm}$ above the two levers to lower the chamber's height and thus reduce the frequency of rearing responses. After this modification, the internal dimensions were $30.5 \times 29.8 \times 15.6 \mathrm{~cm}$. The levers, which required about .2-N force to operate, extended $1.6 \mathrm{~cm}$ into the chamber and were located $6.4 \mathrm{~cm}$ from each sidewall, $12.2 \mathrm{~cm}$ above the grid floor.

For all animals, one of two levels of chamber illumination served to differentiate one signal condition from the other. A .1-A 14.4-V dc overhead lamp produced the brighter of the two levels, while a shielded (white lens cap) .1-A 14.4-V dc light located above each lever produced the dimmer level of illumination. A noise generator (Grason-Stadler Model 901B) delivered $82-\mathrm{dB}$ white noise to a $5.7-\mathrm{cm}$ speaker located below the right lever. This noise stimulus was either pulsed or continuous and served as the signal in both conditions (described below). A shock source (Grason-Stadler Model E 1064) delivered .5-sec $1.0-\mathrm{mA}$ scrambled electric shock to the front and rear walls, levers, and floor (which consisted of .3-cm stainless steel bars), spaced $1.3 \mathrm{~cm}$ apart (center to center). All stimulus conditions and response contingencies were controlled by a minicomputer (Digital Equipment Corporation, PDP-8/E).

\section{Procedure}

During each 100-min session, shock was delivered according to a variable-time 1-min schedule which was independent of the subject's behavior and the stimuli associated with the signal conditions. The shock schedule, which consisted of 100 randomly distributed intershock intervals (range $=11-310 \mathrm{sec}$ ), was based on a constant-probability formula (Catania \& Reynolds, 1968).

At any moment during an experimental session, a subject was in one of two mutually exclusive conditions. All shocks occurring in one of the two conditions were preceded by a 5-sec white-noise $(82-\mathrm{dB})$ stimulus, and the stimulus was presented only prior to shock delivery. This condition is referred to as the reliable-signal condition, since the signal contained reliable information about the occurrence of shock as well as the time of shock delivery. In this condition, the 5 -sec signal could be pulsed $(1 / 2 \sec$ on, $1 / 2 \sec$ off) or continuous (counterbalanced across groups), but the nature of the stimulus was always opposite to the one used in the unreliable-signal condition, which differed for two groups of animals. 
Unreliable information about the occurrence of shock. Half of the subjects (Group A) had the option of choosing between the reliable-signal condition described above and an unreliablesignal condition, in which the probability of the signal terminating in shock was less than one. For these animals all shocks that occurred in the unreliable condition were preceded by the 5 -sec white-noise stimulus. However, additional identical 5-sec noise stimuli that did not terminate in shock were programmed to occur in this condition. The percentage of "extra" signals that could occur if an animal remained in this condition for an entire session was manipulated at different stages of the experiment (Figure 1, top panel). In the uireliable-signal condition, then, the probability of shock in the presence of the signal was less than unity, but, because of the fixed temporal relationship between the signal's onset and the occurrence of shock, information about the time of shock delivery (when shock occurred) was a dependable feature of the condition.

Unreliable information about the time of shock delivery. The other half of the animals (Group B) were permitted to choose between the reliable-signal condition described earlier and an unreliable-signal condition in which information about the time of shock delivery was degraded. For these subjects all shocks that occurred in the unreliable-signal condition were preceded by the white-noise stimulus, and white noise never occurred unless it terminated in shock. Unlike the reliablesignal condition, however, the duration of the noise stimulus was variable (mean $=5 \mathrm{sec}$, range $=1-26 \mathrm{sec}$ ) rather than fixed $(5 \mathrm{sec})$. During the second half of the experiment, the duration of the unreliable signal was made more variable (range = $1-52 \mathrm{sec}$ ) to assess its effect on preference behavior. This manipulation required extending the average duration from 5 to $10 \mathrm{sec}$; to hold the overall amount of discriminable shock-free time constant in both conditions, the duration of the reliable signal was increased to $10 \mathrm{sec}$.

Preference measure (changeover). Subjects were tested every other day for a total of 72100 -min sessions, which were divided into 12 6-session test phases. During each test phase, animals were placed in either the reliable- or unreliable-signal condition (counterbalanced within groups) and permitted to change over to the other condition by emitting a leverpress. The two signal conditions, and, consequently, the changeover options, were reversed every six sessions so that every animal had several opportunities to respond for each condition. Two successive test phases constituted an experimental cycle and represented one opportunity to respond for each condition. To facilitate the initial discrimination, the two signal conditions were reversed every other test day during the first cycle. Shock intensity was gradually increased across the first 12 sessions $(.2-1.0 \mathrm{~mA})$; shock duration, however, remained fixed at $.5 \mathrm{sec}$ throughout the experiment.

When an animal was placed in one signal condition, it always had the option to change over to the other condition. A response on either lever transferred program control to the opposite signal condition for a period of $1 \mathrm{~min}$. Any additional leverpresses during the 1-min changeover interval had no programmed consequences. At the end of $1 \mathrm{~min}$, experimental control automatically returned to the original condition. A $2.5-\mathrm{sec}$ changeover delay, timed from shock onset, was programmed to eliminate changeover responses due to shock-elicited behavior.

The brighter level of chamber illumination (described earlier) identified the reliable- and unreliable-signal conditions equally often for subjects within groups. For any given animal, however, the brighter level of illumination always identified one signal condition, while the dimmer level always identified the other. During the 1-min changeover interval, the animal received the signals and shocks that were preprogrammed to occur for that time period. If the animal happened to respond during a signal, there was an immediate change in chamber illumination, but the signal continued unaffected until it normally timed out or terminated in shock. It is important to note that a leverpress simply substituted the signals used in one condition for those used in the other and in no way modified the shock program. The percentage of a session spent in changeover served as the basic dependent variable.

\section{RESULTS}

The percentage of a session an animal spent in changeover was averaged over the last three sessions of each six-session test phase and then collapsed across subjects within groups. Figure 1 (top panel) shows average percent time in changeover for the animals in Group A. As indicated in Figure 1, no consistent preference for the reliable- or the unreliable-signal condition emerged in any one of the six experimental cycles, even though the unreliable-signal condition contained $100 \%$ extra signals during the last two cycles of the experiment. A repeated-measures analysis of variance with phase, treatment (percentage of possible extra signals), and cycle (within treatment) as factors indicated that there were no significant differences for either the main effects or their interactions $(p>.05)$. The only consistent finding was that the subjects tended to respond for
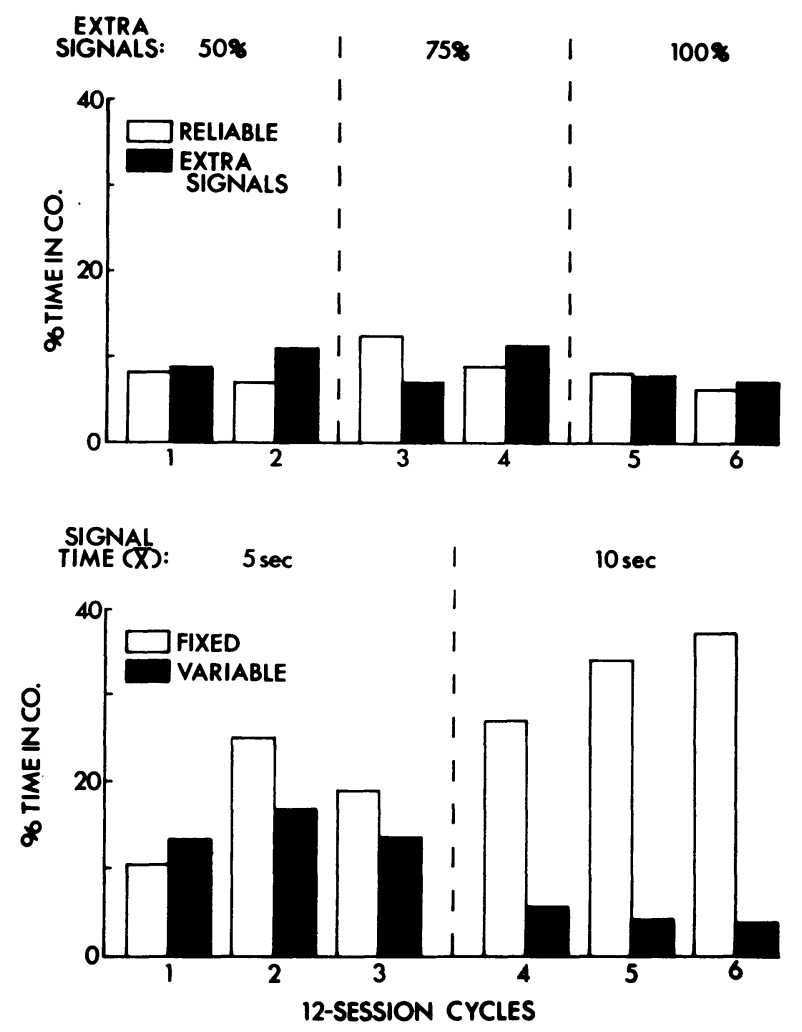

Figure 1. Top panel: Mean percentage of time animals of Group A spent in changeover (CO) when a leverpress produced the reliable- or the unreliable- (EXTRA) signal condition for a 1-min period. The percentage of extra signals that could occur in the unreliable-signal condition are as indicated. Bottom panel: Mean percentage of time animals of Group B spent in the fixed(reliable) and variable- (unreliable) signal conditions. The average duration of the signal is as shown. 
the dimmer level of illumination regardless of the condition that it served to identify.

In contrast to the above findings, subjects in Group B developed a strong preference for the reliable-signal condition (Figure 1, bottom panel). An analysis of variance similar to the one described above revealed a significant main effect for phase $[F(1,33)=22.45$, $\mathrm{p}<.001]$ as well as a significant Phase by Treatment interaction $[\mathrm{F}(1,33)=12.38, \mathrm{p}<.005]$, which indicates that subjects not only preferred the reliable-signal condition but also that this preference increased when the variability of the signal was increased (range: $1-26 \mathrm{sec}$ vs. $1.52 \mathrm{sec}$ ). No other main effects or their interactions were significant. Preference for the reliablesignal condition was shown regardless of the level of illumination that served as its correlated stimulus. Higher rates of responding, however, were maintained when the dimmer of the two levels served to identify the reliable condition.

\section{DISCUSSION}

Within the limitations of the signal parameters tested here, the present findings indicate that when the amount of discriminable shock-free time (safe periods) is comparable, information about the time of shock delivery acquires considerable control over changeover behavior, while information about the probable occurrence of shock does not.

The latter data support and extend some recent observations by Badia et al. (1976), who found that preference for the signaled over the unsignaled condition persisted when the dependability of the signal was reduced by programming extra signals that did not terminate in shock. Badia et al. (1976) accounted for their findings by assuming that the dependability of the signal's absence as a nredictor of shock-free time was the important variable controlling preference for signaled shock. The same interpretation can be applied to the results of Group A, inasmuch as the amount of discriminable shock-free time was about the same in both signal conditions. However, the strong preference that developed in Group B for the reliable-signal condition cannot be attributed to differences in either the quantity or the quality of safe periods. This result suggests, instead, that some aspect of the signal-shock relationship maintained the observed preference behavior.

Perhaps the most obvious explanation as to why subjects in Group B preferred fixed-over variable-duration signals is that the fixed-duration signal contained an implicit safe period, in that shock never occurred immediately after the signal's onset. While this possibility cannot be discounted, there is evidence to suggest that additional safe periods of this magnitude are not sufficient to maintain responding. Note that subjects in Group A did not prefer the reliable-signal condition even though it contained somewhat more discriminable shock-free time than did its counterpart. A second possibility is that information about the time of shock delivery is itself reinforcing (cf. Collier, 1977), perhaps because it reduces uncertainty about when shock will occur (e.g., Hendry, 1969) or because it enables the animals to prepare more effectively for its delivery (e.g., Perkins, 1968). Whatever the explanation turns out to be, the results of Group B suggest that signals in a signaled-shock situation do more than simply divide the animal's time into safe (signal absent) and unsafe (signal present) periods, as has been suggested by some investigators (e.g., Badia, Culbertson, \& Lewis, 1971; Seligman, Maier, \& Solomon, 1971).

\section{REFERENCES}

Arabian, J. M., \& Desiderato, O. Preference for signaled shock: A test of two hypotheses. Animal Learning \& Behavior, 1975, 3, 191-195.

Badia, P.. Culbertson, S., \& Lewis, P. The relative aversiveness of signaled vs. unsignaled avoidance. Journal of the Experimental Analysis of Behavior, 1971. 16. 113-121.

Badia, P., Harsh, J., Coker, C. C., \& Abbott, B. Choice and the dependability of stimuli that predict shock and safety. Journal of the Experimental Analysis of Behavior. 1976. 26. $95-111$.

Catania, A. C.. \& Reynolds, G. S. A quantitative analysis of the responding maintained by interval schedules of reinforcement. Journal of the Experimental Analysis of Behavior, 1968, 11, 327-383.

Collier. A. C. Preference for shock signals as a function of the temporal accuracy of the signal. Learning and Motivation, 1977, 8, 159-170.

HENDRY, D. P. Reinforcing value of information: Fixed ratio schedules. In D. P. Hendry (Ed.), Conditioned reinforcement. Homewood, Ill: Dorsey, 1969.

Perkins, C. C., JR. An analysis of the concept of reinforcement. Psychological Review, 1968, 75, 155-172.

Seligman. M. E. P., Maier, S. F., \& Solomon, R. L. Unpredictable and uncontrollable aversive events. In F. R. Brush (Ed.), Aversive conditioning and learning. New York: Academic Press, 1971.

(Received for publication June 28, 1977.) 